\title{
Non-Parametric Model of Magneto-Rheological (MR) Damper Based on Adaptive Neuro-Fuzzy Inference System (ANFIS)
}

\author{
Ling Zheng ${ }^{1, a}$, Fei Liu ${ }^{1, b}$, Zhongyong Zhou ${ }^{1, \mathrm{c}}$ \\ ${ }^{1}$ State Key Laboratory of Mechanical Transmission, Chongqing University, Chongqing, 400044, \\ China

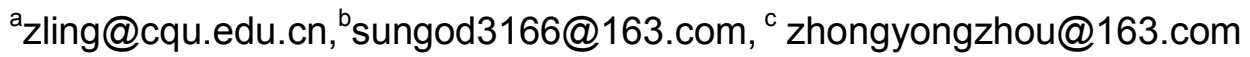

Keywords: magneto-rheological (MR) damper, non-parametric model, adaptive neuro-fuzzy inference system (ANFIS)

\begin{abstract}
Magneto-rheological (MR) damper is a typical non-linear actuator. The nonlinear relationship between the input and the output of MR damper should be characterized accurately in order to produce a required control action supplied by MR damper. The challenges of the conventional parametric model are the time-consuming and the requirement of complex parameter identification for MR damper. In this paper, a non-parametric model of MR damper based on the adaptive neuro-fuzzy system theory is presented to overcome the drawbacks of the conventional parametric model. This non-parametric model is developed by means of two adaptive neural fuzzy sub-systems which are designed to describe the nonlinear relationship in MR damper successfully. One sub-system is used to characterize the dependence of the damping force on velocity and acceleration, the other sub-system is used to characterize the dependence of the damping force level on the control voltage. The proposed non-parametric model is identified by experimental results. Furthermore, the accuracy of the model is investigated and evaluated. The model supplies a key technical support to achieve excellent control performances for semi-active suspension systems with MR dampers in vehicle.
\end{abstract}

\section{Introduction}

In the past decade, magneto-rheological (MR) dampers have been developed as one of the most promising devices in vibration control applications due to their mechanical simplicity, wide dynamic range, low power consumption and high force supply as well as robustness for external excitation. [1].They have been considered as efficient devices for vibration suppression in civil, aerospace and automobile engineering.

A model that can predict the hysteresis force of the MR damper is required to take advantage of the exceptional characteristics of MR dampers. There have been many efforts devoted to model the non-linear behavior of the MR damper. These models are classified as the quasi-static and dynamic models. Although the quasi-static models are capable of describing force-displacement behavior of MR dampers reasonably well, they are not sufficient to describe the nonlinear force-velocity behavior of these dampers [2].

Many dynamic models have been developed to overcome this shortcoming. These models include parametric and non-parametric models. The Bouc-Wen model is a typical of parametric model, which has been used extensively by different researchers to simulate the hysteresis phenomenon of MR dampers. However it is not easy to identify all parameters accurately and perfectly. Later, Spencer et al [3] proposed a phenomenological model for MR dampers based on the Bouc-Wen model. This model is able to capture the force roll-off in the low velocity region that is observed in the experimental data. However, in Spencer's model, many parameters need to be estimated as in Bouc-Wen model. Wang et al [4,5] developed the equivalent characteristic method, which considers the relationships between the hysteresis phenomenon and the frequency and stroke of the excitation as well as the current. The hysteresis force/velocity model is established by symmetrical sigmoid function. 
Another class of dynamic models mentioned above are non-parametric models. In general, non-parametric models are more robust and flexible than parametric models. They could also be more effectively used for plant modeling in practical control applications. Chang and Roschke [6] proposed a non-parametric model using multi-layer perception neural network integrated optimization method for a satisfactory representation of damper behavior. Wang and Liao [1,7] explored the modeling of MR dampers by using a trained direct identification based on recurrent neural network. The model could predict the dynamic responses of these dampers in high precision, however, its architecture and training method are still complex.

Wang et al [8] proposed a non-parametric model based on fuzzy logic theory. In the model, the feature of MR dampers in the individual voltage could be captured successfully. The disadvantage of the model is that no variation of voltage is involved. Schurter et al [9] developed a model by means of an adaptive neuro-fuzzy inference system. The model can be used to model the nonlinear characteristics of MR dampers well. However, too many fuzzy membership functions are applied. Kyoung et al [10] explored the non-parametric modeling of MR dampers by a self tuning fuzzy system (STF) based on neural technique. The designed STF model contains two parts: one is the neural-fuzzy inference (NFI) and the other is the scheduling gain fuzzy inference (SGFI). Although it is effective to model the nonlinear behavior of MR dampers, no methodology is given about how these fuzzy inference rules can be formulated.

In this paper, a novel non-parametric model based on adaptive neuro-fuzzy inference system (ANFIS) approach is presented. This model is an extension of STF model [10]. A neural network mimics the biological information processing mechanisms. It is typically designed to perform a nonlinear mapping from a set of inputs to a set of outputs. Hence, the advantage of neural network is used to train and form the inference rules of fuzzy systems, and it avoid that there is no systematic method to design and examine the number of rules, input space partitions and membership functions (MFs). The input variables of the non-parametric model are the piston rod velocity, acceleration and the voltage applied to the damper coil. And the output is damping force of MR damper. Through the comparison with reference model, this neuro-fuzzy approach provides a means of training a family of membership functions to achieve an accurate modeling for the behavior of MR damper [11], and the designed fuzzy inference system has higher learning ability that improves the identification quality. As the result, the non-parameterization model has better accuracy.

\section{Non-parametric model}

The experimental data from a MR damper (RD-1005-3) manufactured by Lord Corporation is used to demonstrate and test the accuracy of the proposed model in this paper. The nonlinear relationship between the input and the output of the MR damper can be given [12]:

$$
F=f_{0}+C_{b} \dot{x}+\frac{2}{\pi} f_{y l} \tan ^{-1}\left[k\left(\dot{x}-\dot{x}_{0} \operatorname{sgn} \ddot{x}\right)\right]
$$

where,

$$
\begin{gathered}
C_{b}=C b /\left(1+10.34 e^{-1.04 v}\right) \\
f_{y l}=f_{y} /\left(1+e^{-1.1(v-2.3)}\right) \\
\dot{x}_{0}=C w /\left(1+1.81 e^{-0.2 v}\right)
\end{gathered}
$$

$f_{0}$ is the offset force; $C b$ is the slope coefficient of hysteretic curve; $f_{y}, k$ are the coefficients which relate to the maximum damping force; $C w$ is the width coefficient of hysteretic curve, $v$ is control voltage which can be changed from $0 \sim 7.5 v$. 


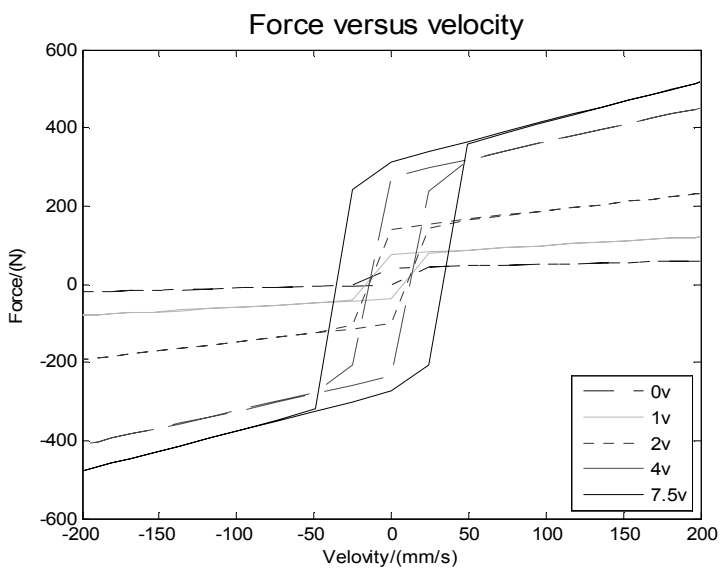

Fig. 1 Force versus velocity of MR damper (RD-1005-3)

Above model is a typical of parametric model which demonstrates nonlinear characteristics of the MR damper. Figure1 is experimental results of MR damper (RD-1005-3). A set of parameters can be identified in order to characterize the performance of MR damper (RD-1005-3) by using above parametric model. In this paper, a non-parametric model for MR damper (RD-1005-3) is developed by using neural network and fuzzy logic methods to capture nonlinear characteristics of MR damper (RD-1005-3).

Architecture of Non-Parametric Model. As well known, the damping force of MR damper depends on the displacement, velocity as well as the voltage applied for the coil in MR damper. It is not easy to describe the nonlinear relationship of them. In order to simplify and describe this relationship clearly, two sub-systems are designed. They are ANFIS I and ANFIS II . ANFIS II is used to characterize the dependence of the damping force $(f)$ on velocity and acceleration. ANFIS I is used to characterize the dependence of the damping force level $(K)$ on the control voltage. Consequently, the actual damping force should be a product of the damping force $(f)$ from ANFIS II and the damping force level $(K)$ from ANFIS I . It is given by:

$F=K \times f$

Therefore, the overall scheme of the proposed non-parametric model can be established as shown in Figure2.

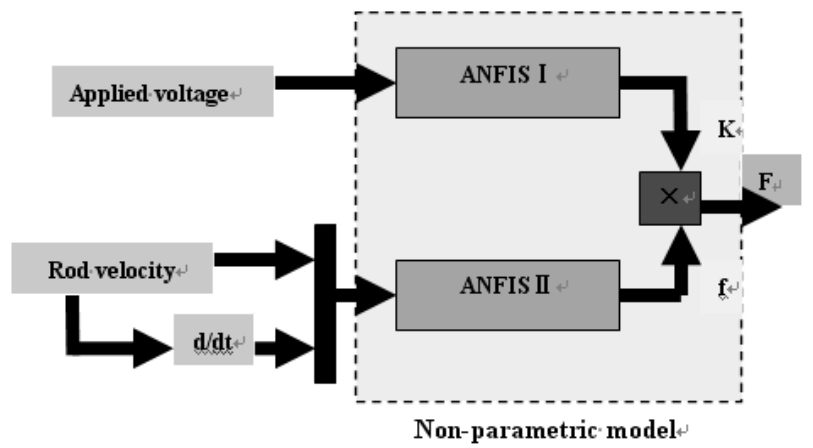

Fig. 2. Architecture of the non-parametric model

Adaptive Neuro-Fuzzy Inference System (ANFIS). A neuro-fuzzy approach is developed to integrate pattern recognition capabilities of neural networks and flexible modeling advantages of fuzzy logic here. Neuro-fuzzy approach can provide a means to train a family of membership functions. ANFIS are a class of adaptive networks that are functionally equivalent to fuzzy inference systems. ANFIS uses a hybrid learning algorithm that combines the back propagation gradient descent and least squares methods to create a fuzzy inference system whose membership functions are iteratively adjusted according to a group of the input and output data predetermined. 
The fuzzy model is based on the first order Takagi-Sugeno (T-S) architecture that is generally composed of $r$ rules:

$$
\text { Rule } i \text { : IF } x_{1} \text { is } A_{i 1} \text { and } x_{2} \text { is } A_{i 2} \text { and } x_{n} \text { is } A_{i n} \text {, THEN } y=b_{i o}+b_{i 1} x_{1}+b_{i 2} x_{2}+\cdots+b_{i n} x_{n}
$$

Where: $x_{1}, x_{2} \ldots x_{n}$ are antecedent variables;

$A_{i 1}, A_{i 2} \ldots A_{i n}$ are fuzzy sets defined over the domains of the antecedents respectively, $y$ is the consequent variable.

A T-S fuzzy model is a computationally efficient platform that can establish nonlinear associations through the piecewise linear relationships. Such a function is used to describe nonlinear nature of MR damper. The architecture of the proposed ANFIS is shown in Fig.3 [13].

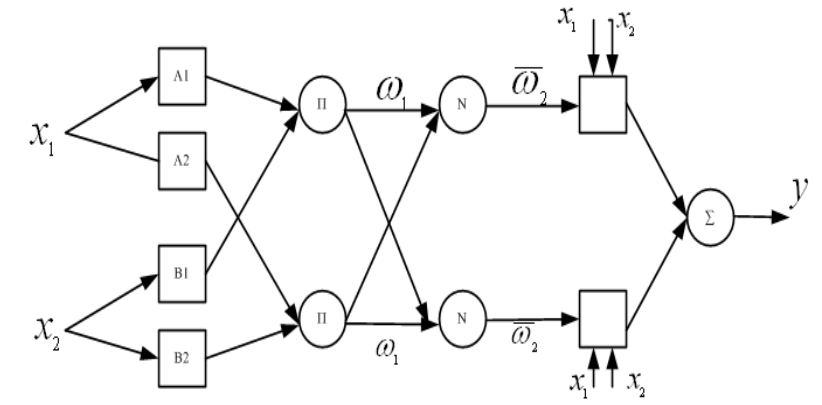

Fig. 3. Architecture of classic ANFIS

Layer 1: Each node in this layer is an adaptive node with a node function:

$$
O_{1, i}=\mu_{A i}\left(x_{1}\right) \quad i=1,2
$$

or

$$
O_{1, i}=\mu_{B(i-2)}\left(x_{2}\right) \quad i=3,4
$$

where, $x_{1}\left(\right.$ or $\left.x_{2}\right)$ is the input node, and $A_{i}\left(\right.$ or $\left.B_{(i-2)}\right)$ is a linguistic label associated with this node, such as "large" or "small". Therefore $O_{1, i}$ is the membership grade of a fuzzy set $A$, and usually typical membership function is bell function.

Layer 2: Each node in this layer is a fixed node labeled $\Pi$ in Fig.3. Its output is the product of all the incoming signals.

$$
O_{2, i}=w_{i}=\mu_{A i}\left(x_{1}\right) \mu_{B i}\left(x_{2}\right) \quad i=1,2
$$

Layer 3: Each node in this layer is a fixed node labeled $N$ in Fig.3. It calculates the ratio of the rule's firing strength to the sum of all rule's firing strengths:

$$
O_{3, i}=\bar{w}_{i}=\frac{w_{i}}{w_{1}+w_{2}} \quad i=1,2
$$

Layer 4: Each node in this layer is an adaptive node with a node function:

$$
O_{4, i}=\bar{w}_{i} f_{i}=\bar{w}_{i}\left(p_{i} x_{1}+q_{i} x_{2}+r_{i}\right) \quad i=1,2
$$

Layer 5: This node in this layer is a fixed node labeled sum, which computes the overall output as the summation of all incoming signals:

$$
O_{5, i}=\sum_{i} \bar{w}_{i} f_{i}=\frac{\sum_{i} f_{i} w_{i}}{\sum_{i} w_{i}}
$$


ANFIS I is a system with single input and single output. It provides a description of scheduling gain fuzzy inference which works as an intelligent switch to tune the dependence of the damping force level $(K)$ on the control voltage. The range of the input voltage is covered from 0 to $7.5 \mathrm{~V}$, and three triangle membership functions, " $\mathrm{Z}$ ", "S", and "B", are considered for the input variable. A T-S fuzzy inference system is created by using the neural networks, whose membership functions are iteratively adjusted according to a set of discrete data from the experimental velocity curve of RD-1005-3.

Before ANFIS I is trained, an initial Fuzzy Inference System (FIS) is formulated. A hybrid learning algorithm is used to identify parameters of FIS. It applies a combination of the least-squares method and the back propagation gradient descent method to train FIS membership function parameters. ANFIS I can also be invoked by using an optional argument for model validation. This type of model validation that takes place with this option is a checking for model over fitting, and the argument is a data set called the checking data set. The structure of ANFIS I after training is shown in Fig.4.

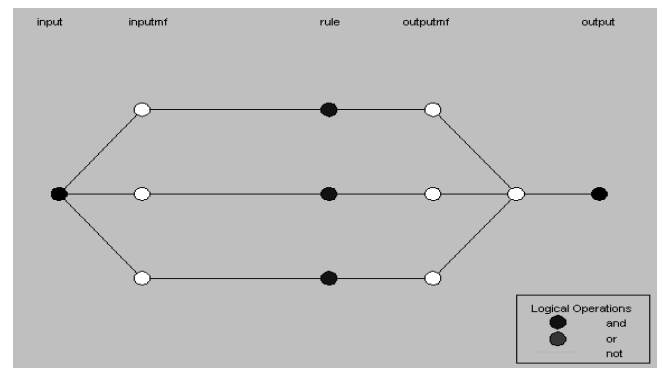

Fig. 4. Structure of ANFIS I after training

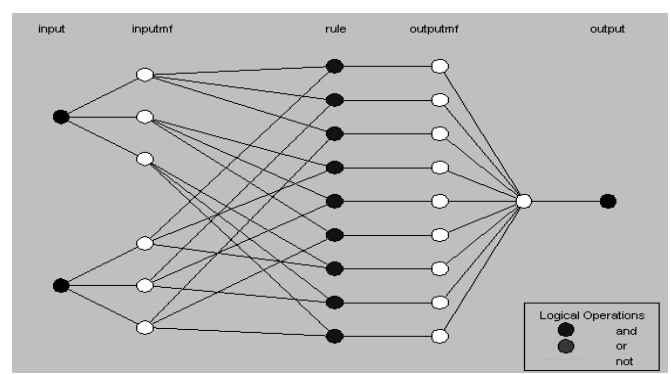

Fig. 5. Structure of ANFIS II after training

The ANFIS II is designed to estimate the dependence of the damping force of MR damper on velocity and acceleration. Therefore, it has two inputs (velocity and acceleration) and one output (the damping force). For each input variable, three triangle membership functions with "nb", "ze" and "pb" are considered, whose initial input membership functions are bell function. Expression of typical bell function is shown as follows:

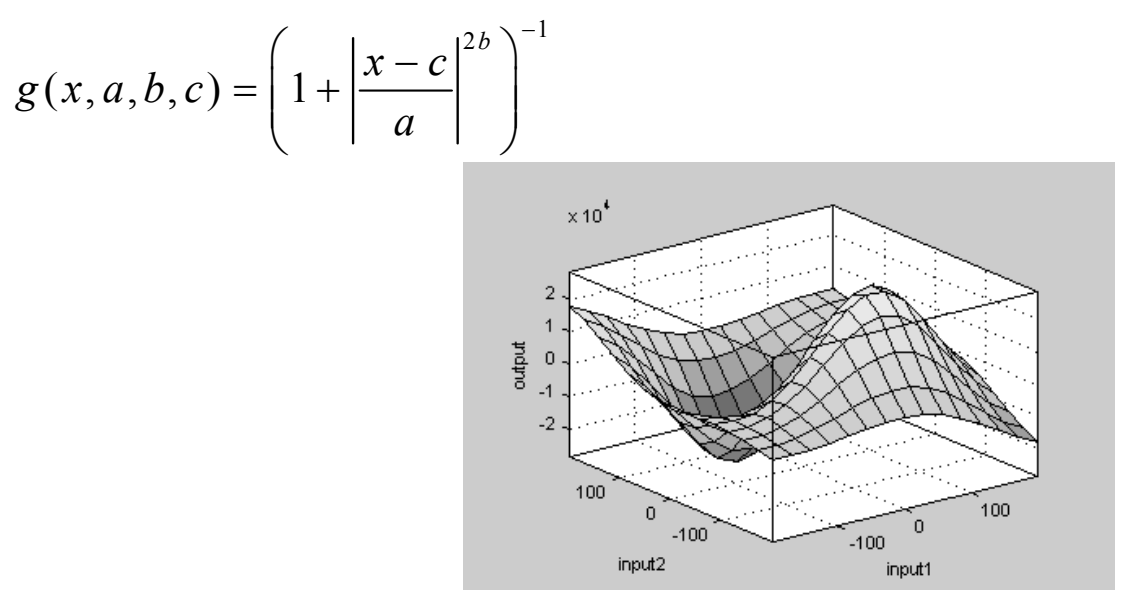

Fig. 6. Inference surface of ANFIS II after training

The structure of ANFIS II after training is shown in Fig.5. Fuzzy inference surface is illustrated in Fig.6. It can be seen that the surface after training is continuous and smooth.

\section{Results and comparisons}

The proposed non-parametric model based on Adaptive Neuro-Fuzzy Inference System (ANFIS) is used to characterize the behavior of MR damper (RD-1005-3) manufactured by Lord Corporation. The performance of RD-1005-3 is demonstrated in Fig.1. The results are compared graphically and numerically with the curves in Fig. 1 to validate the accuracy of this proposed model. The data in Fig. 1 is used to train and identify the presented ANFIS model. 
A quantitative error analysis is proposed to evaluate normalized model errors [14] as follows:

$$
E=1-\frac{\sum_{k=1}^{n}|f(t k)-f(t \hat{k})|}{n \max (|f(t k)|)}
$$

where, $\mathrm{E}$ is relative approximating precision of the model, $\mathrm{n}$ is the number of discrete nodes, $f(t k)$ is the damping force obtained by experiment, and $f(t \hat{k})$ is the damping force predicted by the ANFIS model.

The comparison results are demonstrated in Fig. $7 \sim$ Fig. 14 and the dash line is from the proposed model and the solid line is from the experimental data.

Fig.7 Fig.10 illustrate the velocity characteristics of MR damper (RD-1005-3). It can be seen clearly that two curves can coincide each other in low and high velocity regions. However, the coincidence in transition region between low velocity hysteretic region and high velocity linear region is not satisfactory enough. Therefore, the modeling accuracy of the proposed model in transition region needs to be improved by training this model more times or modify the fuzzy membership rules in details.

Fig.11 Figure14 show the displacement characteristics of MR damper (RD-1005-3). It is clear that the results from the model can coincide with experimental data in almost all regions. This means that the model can match the displacement characteristics of MR damper (RD-1005-3) perfectly.

Above results demonstrate that the nonlinear characteristics of MR damper (RD-1005-3) can be estimated in a relative high accuracy by the proposed non-parametric model.

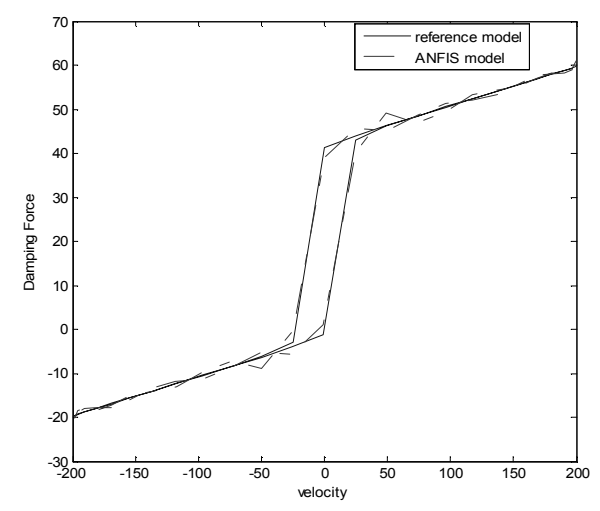

Fig. 7. Force versus velocity when $v=0 \mathrm{~V}$

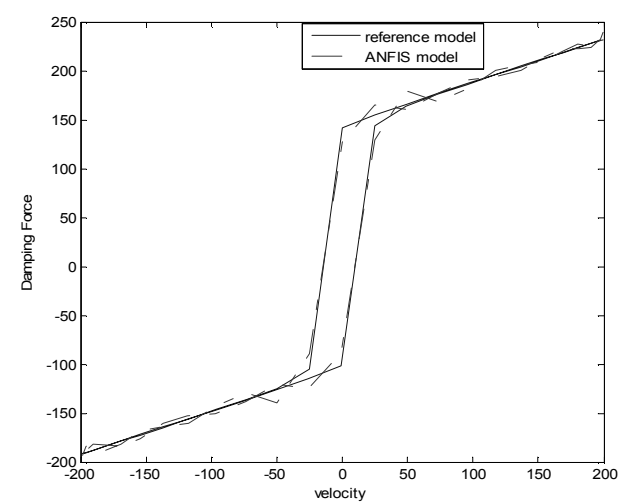

Fig. 9. Force versus velocity when $v=2 \mathrm{~V}$

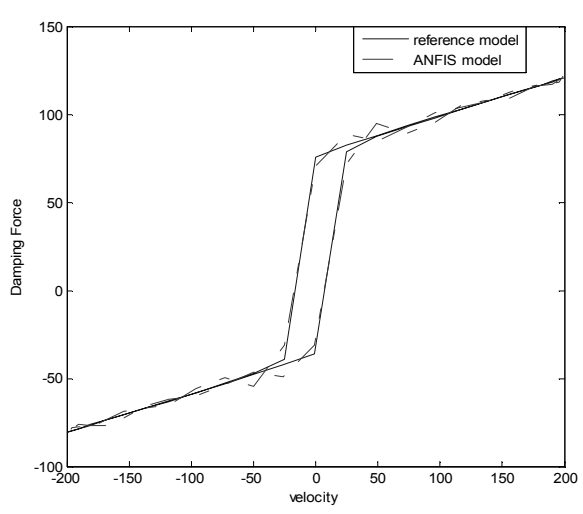

Fig. 8. Force versus velocity when $v=1 \mathrm{~V}$

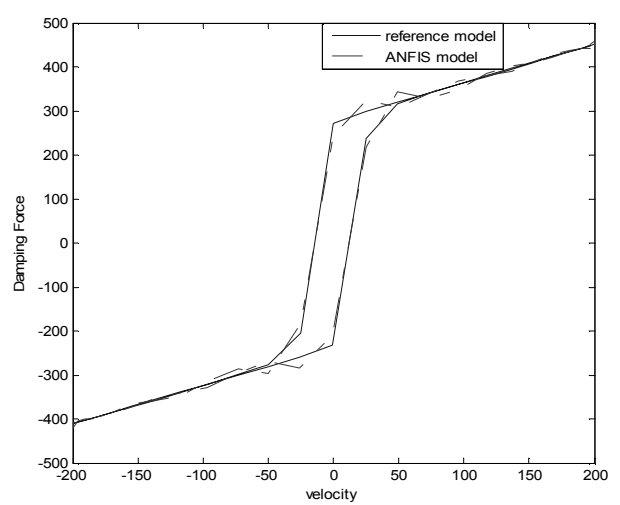

Fig. 10. Force versus velocity when $v=4 \mathrm{~V}$ 

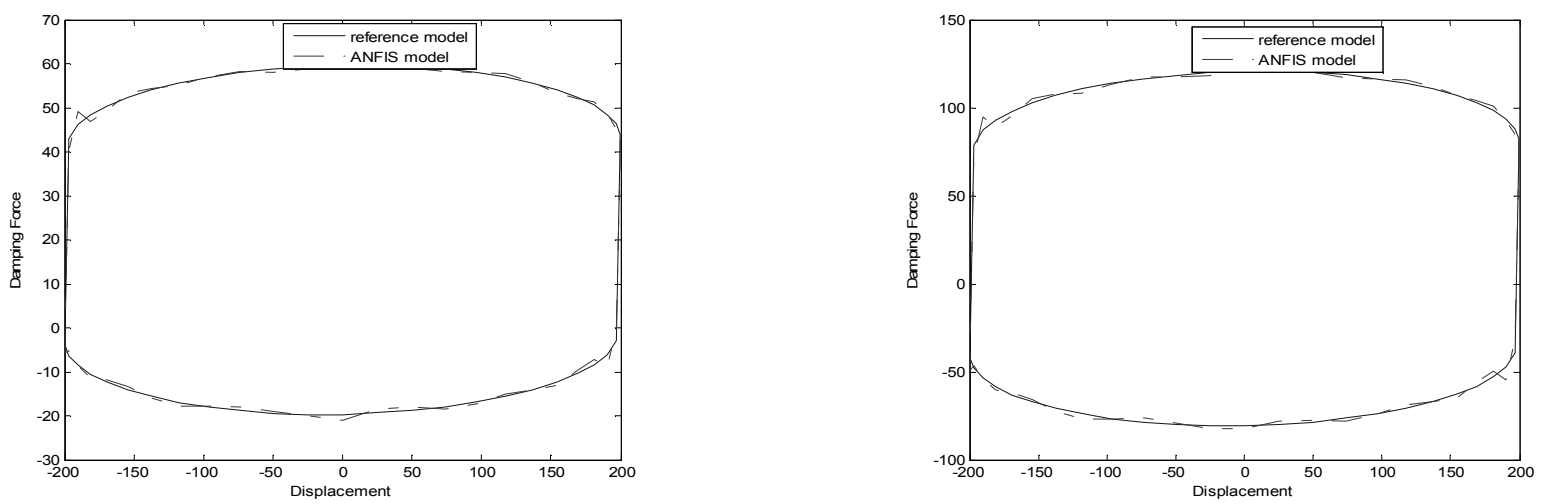

Fig. 11. Force versus displacement when $v=0 \mathrm{~V}$. Fig. 12. Force versus displacement when $v=1 \mathrm{~V}$
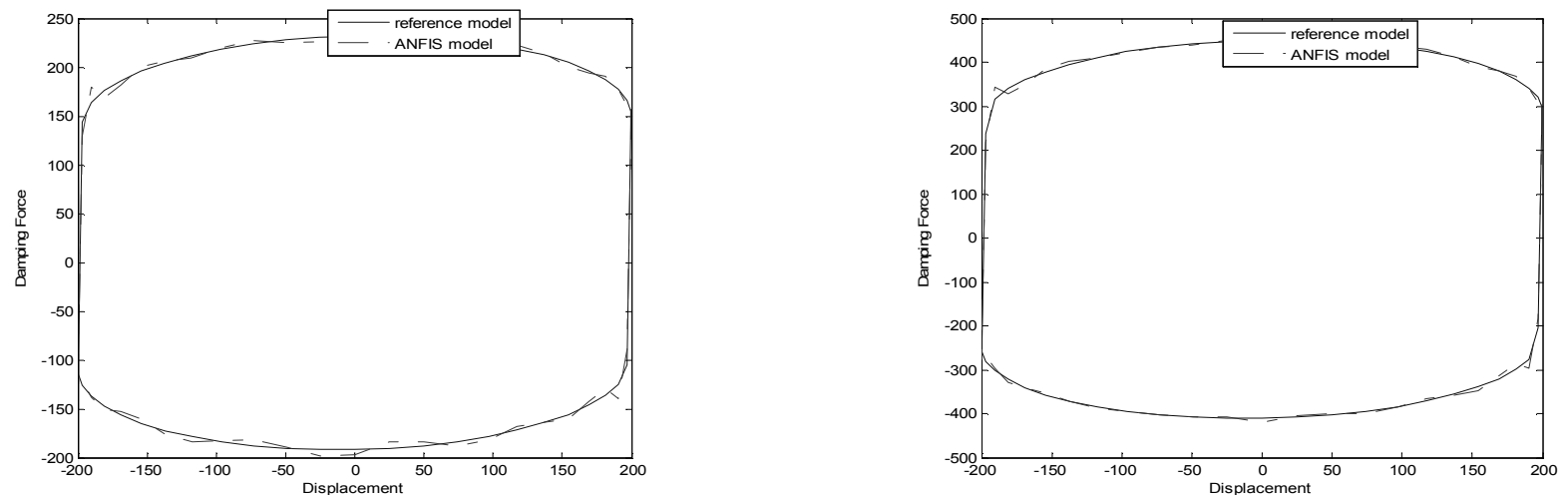

Fig. 13. Force versus displacement when $v=2 \mathrm{~V} \quad$ Fig. 14. Force versus displacement when $v=4 \mathrm{~V}$

Table1 Approximation accuracy of Non-parametric model

\begin{tabular}{cc}
\hline voltage/V & accuracy \\
\hline 0 & $98.46 \%$ \\
1 & $98.06 \%$ \\
2 & $97.73 \%$ \\
4 & $98.29 \%$ \\
\hline
\end{tabular}

Equation (13) is used to evaluate the error between the presented model and the experimental data. The results are listed in Table1. Table1 shows that when the applied voltages are changed, an excellent agreement between the ANFIS model and the experimental data can be achieved. The maximum error is only $2.77 \%$. Although this model looks a little bit complex in architecture, in fact, the process of training and identification is relatively simple. On the other hand, it can approach the actual test results in a high accuracy. Therefore, the non-parametric model should be available to be used in a real vibration control system instead of conventional parametric models.

\section{Conclusion}

A simple and direct modeling method for MR damper by using the ANFIS mechanism has been proposed and developed. The experimental model of the RD-1005 MR damper is considered as the target of modeling. The proposed model can accurately capture the inherent hysteretic behavior of MR damper and does not require too much computation cost as conventional parametric models require. As a black box model, it is not necessary to understand the internal structure of MR damper in details. The training of the non-parametric model only depends on the experimental data. This means that the model can also be used to characterize nonlinear hysteretic behavior of some smart devices beside typical MR dampers. The identification of the model shows that it can characterize the hysteretic behavior of MR damper in enough accuracy. Therefore, the model demonstrates a potential application in practical semi-active vibration control system with MR dampers. 


\section{Reference}

[1] Wang, D.H. and Liao, W.H. 2005. "Modeling and Control of Magneto-Rheological Fluid Damper Using Neural Networks," Smart Materials and Structure, 14(1):111-126.

[2] Yang, G. 2001. "Large-scale Magnetorheological Fluid Damper for Vibration Mitigation: Modeling, Testing and Control," PhD Dissertation University of Notre Dame, IN.

[3] Spencer, B.F., Dyke, S.J. and Sain, M.K. 1997. "Phenomenological Model for Magneto-rheological Dampers," Journal of Engineering Mechanics, 123(3): 230-238.

[4] Wang, E.R., MA, X.Q. and RAKHELA, S. 2003. "Modeling the Hysteretic Eharacteristics of a Magnetorheological Fluid Damper," Journal of Automobile Engineering, 217(D7):537-550.

[5] Wang, E.R. 2005. "Syntheses and Analyses of Semi-active Control Algorithms for a Magneto-rheological Damper for Vehicle Suspensions," Montreal: Concordia University .

[6] Chang, C.C. and Roschke, P. 1998. "Neural Network Modeling of a Magnetorheological Damper," Journal of Intelligent Material Systems and Structures, 9(9):755-764.

[7] Wang, D.H. and Liao, W.H. 2001. "Neural Network Modeling and Controllers for Magneto-rheological Fluid Dampers," Proc of 10th IEEE Int. Conf. on Fuzzy Systems, 1323-1326.

[8] Wang, X.H. and Zhang, P.Q. 2000. "Fuzzy-Logical Non-parameter Modeling of MR Damper," Journal of University of Science and Technology of China, 30(2): 218-222.

[9] Schuler, K.C. and Roschke, P.N. 2000. "Fuzzy Modeling of a Magneto-rheological Damper Using ANFIS," Proceedings of 9th IEEE international Conference on Fuzzy Systems, San Antonio, TX, 1: 122-127.

[10] Ahn, K.K., Truong, D.Q. and Islam, M.A. 2009. "Modeling of a Magneto-rheological (MR) Fluid Damper Using a Self Tuning Fuzzy Mechanism," Journal of Mechanical Science and Technology, 23: 1485-1499.

[11] Jang, J.S., Sun, C.T. and Mizutani, E. 1997. "Neuro-fuzzy and Soft Computing: a Computational Approach to Learning and Machine Intelligence," Prentice Hall, Inc., Upper Saddle River, NJ.

[12] Weng, J.Sh., Hu, H.Y. and Zhang, M.K. 2000. "Experimental Modeling of Magneto-Rheological Dampers," Journal of Vibration Engineering, 13(4): 616-621.

[13][13] Jyh, Sh.R.J. 1993. “ANFIS:Adaptive-Network-Based Fuzzy Inference System,” IEEE Transactions on Systems, Man and Cybernetics, 23(3):665-685.

[14] Wang, H. and Hu, H.Y. 2006. "The Fuzzy Approximation of MR Damper," Journal of Vibration Engineering, 19(1): 31-36. 